Cite this article as: Frick $A E$, Nackaerts $K$, Moons J, Lievens $\mathrm{Y}$, Verbeken $\mathrm{E}$, Lambrecht $\mathrm{M}$ et al. Combined modality treatment for malignant pleural mesothelioma: a single-centre long-term survival analysis using extrapleural pneumonectomy. Eur J Cardiothorac Surg 2018; doi:10.1093/ejcts/ezy385.

\title{
Combined modality treatment for malignant pleural mesothelioma: a single-centre long-term survival analysis using extrapleural pneumonectomy
}

Anna Elisabeth Frick ${ }^{a, b, *}$, Kristiaan Nackaerts ${ }^{b, c}$, Johnny Moons ${ }^{a, b}$, Yolande Lievens ${ }^{d}$, Eric Verbeken ${ }^{\text {e,f }}$, Maarten Lambrecht ${ }^{\mathrm{g}, \mathrm{h}}$, Johan Coolen ${ }^{\mathrm{f}, \mathrm{i}}$, Christophe Dooms ${ }^{\mathrm{b}, \mathrm{c}}$, Johan Vansteenkiste ${ }^{\mathrm{b}, \mathrm{c}}$, Paul De Leyn ${ }^{\mathrm{a}, \mathrm{b}}$ and Philippe Nafteux ${ }^{\mathrm{a}, \mathrm{b}}$; for the Leuven Lung Cancer Group

a Department of Thoracic Surgery, University Hospitals KU Leuven, Leuven, Belgium

b Department of Chronic Disease, Metabolism and Ageing, KU Leuven, Leuven, Belgium

c Department of Respiratory Diseases, Respiratory Oncology Unit, University Hospitals KU Leuven, Leuven, Belgium

d Department of Radiation Oncology, Ghent University Hospital, Ghent, Belgium

e Department of Histopathology, University Hospitals KU Leuven, Leuven, Belgium

f Department of Imaging and Pathology, KU Leuven, Leuven, Belgium

g Department of Radiation Oncology, University Hospitals KU Leuven, Leuven, Belgium

h Department of Oncology, KU Leuven, Leuven, Belgium

Department of Radiology, University Hospitals KU Leuven, Leuven, Belgium

* Corresponding author. Department of Thoracic Surgery, University Hospitals KU Leuven, Herestraat 49, 3000 Leuven, Belgium. Tel: +32 16340234 ; fax: +32 16 34 68 21; e-mail: annaelisabeth.frick@uzleuven.be (A.E. Frick).
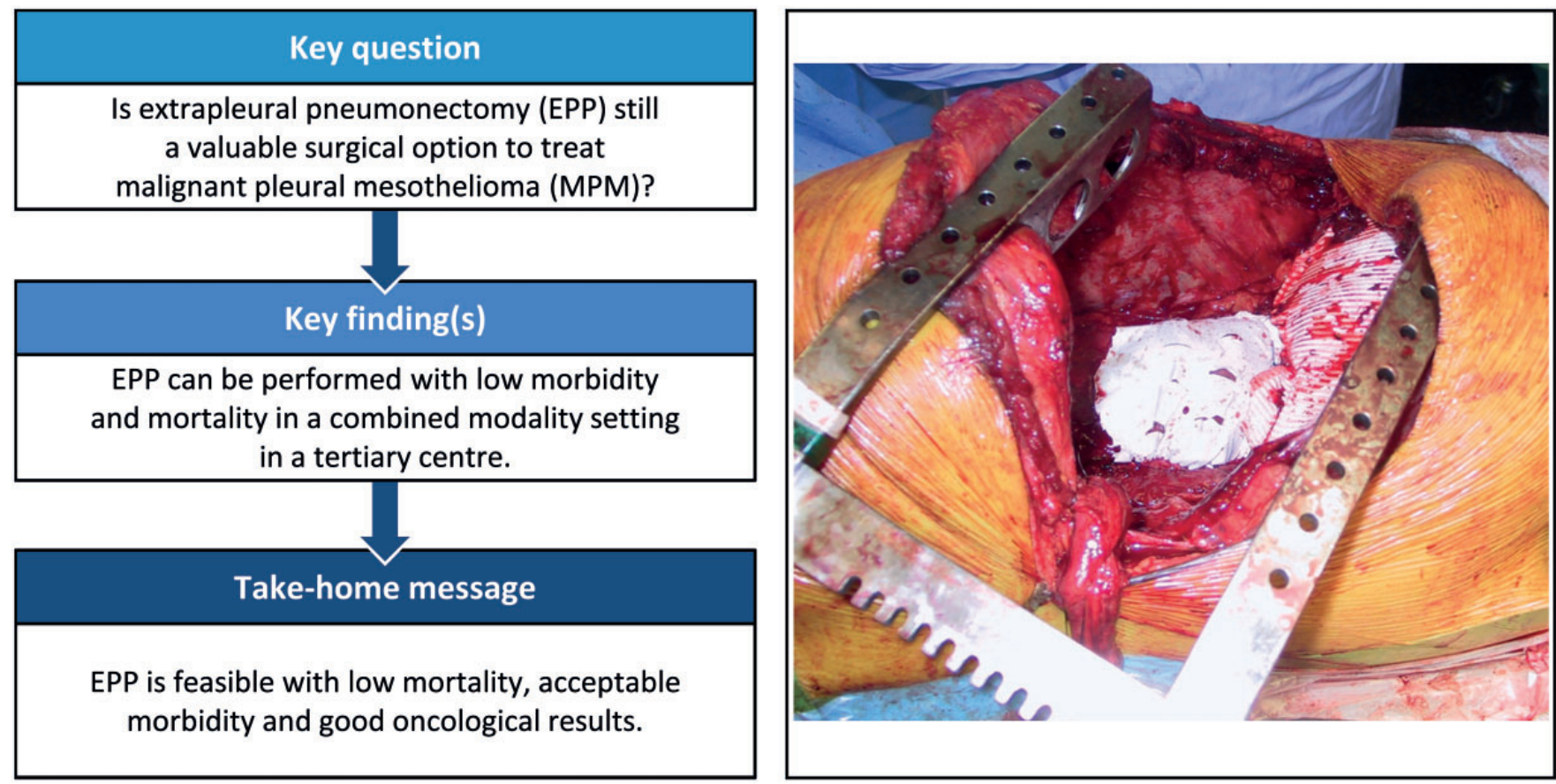


\begin{abstract}
OBJECTIVES: Combined modality treatment (CMT) for malignant pleural mesothelioma (MPM) remains a matter of debate regarding the choice of surgical procedure: extrapleural pneumonectomy (EPP) or pleurectomy/decortication.
\end{abstract}

METHODS: We performed a prospective interventional cohort study between 2003 and 2014. All consecutive patients with any histological MPM subtype, $\leq 70$ years old, World Health Organization performance status $\leq 1$, medically fit for pneumonectomy and stage cT1-2cN02cM0 (TNM7) or lower were included. Eligibility for CMT was discussed by the multidisciplinary tumour board. Our local CMT protocol consisted of induction chemotherapy, followed by EPP and hemithoracic radiotherapy. Induction chemotherapy consisted of 3 cycles of cisplatin $\left(75 \mathrm{mg} / \mathrm{m}^{2}\right.$ day 1$)$ and pemetrexed $\left(500 \mathrm{mg} / \mathrm{m}^{2}\right.$ day 1$)$, each administered once every 3 weeks. If non-progressive, EPP was performed followed by hemithoracic radiotherapy (most frequently, intensity-modulated radiotherapy; dose $54 \mathrm{~Gy} / 1.8 \mathrm{~Gy} \pm$ boost). Feasibility and long-term survival analyses were performed. Overall survival and disease-free survival (DFS) were calculated from histological confirmation of a diagnosis of MPM.

RESULTS: Out of 197 patients, 97 started with CMT (79 epithelioid, 15 mixed and 3 sarcomatoid tumours, based on histological analysis). Clinical TNM was IA $(n=9) / I B(n=8) / I I(n=57) / I I I(n=23)$. A total of 76 patients underwent surgery (EPP: $n=56$; exploratory thoracotomy: $n=20$ ). The in-hospital mortality rate was $3.6 \%$. Out of 56 patients who underwent surgery, 47 completed the entire CMT protocol. The intent-to-treat median and 5-year OS were 22.4 [95\% confidence interval $(\mathrm{Cl})=15.5-27.9]$ months and $11.2 \%(95 \% \mathrm{Cl}=6.9-23.4)$. In patients who completed the CMT protocol $(n=47)$, these values were $33.2(95 \% \mathrm{Cl}=23.0-45.0)$ months and $24.2 \%(95 \% \mathrm{Cl}=13.4-43.8)$. The intent-to-treat median and 5-year DFS were $15.6(95 \% \mathrm{Cl}=14.0-17.3)$ months and 9.9\% $(95 \% \mathrm{Cl}=5.1-19.2), 19.8(95 \% \mathrm{Cl}=16.8-27.7)$ months and $17.2 \%(95 \% \mathrm{Cl}=8.6-34.1)$ in those who had the full CMT. The Cox proportional hazards model showed a significantly lower DFS in positive lymph nodes (HR 2.79, 95\% $\mathrm{Cl}=1.35-5.78 ; P=0.006)$. In 30 (64\%) patients with epithelioid type MPM without positive lymph nodes (pN0) after EPP, the 5-year DFS was 27.0\% (95\% Cl=14.1-51.7).

CONCLUSIONS: CMT with EPP for MPM is feasible, with an acceptable surgical mortality rate, and results in a 5-year survival rate of $24 \%$. Careful patient selection (staging and physical performance) is extremely important.

Keywords: Malignant pleural mesothelioma $\cdot$ Extrapleural pneumonectomy $\cdot$ Combined modality treatment

\section{INTRODUCTION}

Malignant pleural mesothelioma (MPM) is a rare, slowly growing tumour with a poor prognosis. According to the Belgian national cancer registry, MPM accounted for $0.6 \%$ of cancer diagnoses in men and $0.2 \%$ in women in 2008 . The 1 -year relative survival rate in men was $46.3 \%$ and in women, $50.7 \%$, whereas the 5 -year relative survival results were very poor, i.e. $4.9 \%$ and $8.3 \%$ respectively [1].

The optimal treatment of MPM has been a continuous matter of debate. Surgery-based combined modality treatments (CMTs) seem to confer the best chances of long-term survival in selected patients with MPM. In this context, 2 surgical techniques, extrapleural pneumonectomy (EPP) and radical pleurectomy/decortication, are mostly proposed when a macroscopic complete resection seems achievable in highly selected cases.

The last decade has witnessed the increased use of pleurectomy/decortication [2, 3], especially after publication of the results of the randomized MARS (Mesothelioma and Radical Surgery) trial, which showed no advantage of EPP compared to chemotherapy alone [4-7]. However, this trial has been highly criticized for its small size and high postoperative rates of morbidity and mortality [8]. In contrast, several large series from experienced centres have shown promising results with low rates of morbidity and mortality with EPP-based CMT [9-11].

In 2003, we started using our treatment protocol with EPPbased CMT for eligible patients with MPM. Our goal was to present an analysis of our single-centre MPM cohort to evaluate the feasibility, toxicity and effectiveness of EPP-based CMT in patients with MPM.

\section{MATERIALS AND METHODS}

\section{Study design}

In a prospective, interventional, single-centre cohort study, all patients with MPM diagnosed and treated in our institution from March 2003 through December 2014 were included. Our protocol was approved by the research ethics committee of the University Hospital Leuven, Leuven, Belgium (ML2775), and all patients gave informed consent.

Table 1: Characteristics of all patients diagnosed with MPM and of all patients who were considered for CMT

\begin{tabular}{|c|c|c|}
\hline & $\begin{array}{l}\text { Total number } \\
\text { of patients with } \\
\text { MPM }(\%), N=197\end{array}$ & $\begin{array}{l}\text { Patients } \\
\text { considered for } \\
\text { CMT(\%), N=97 }\end{array}$ \\
\hline \multicolumn{3}{|c|}{ Age at diagnosis (years) } \\
\hline Median & 61.5 & 58.5 \\
\hline Range & $18-86$ & $18-70$ \\
\hline \multicolumn{3}{|l|}{ Gender, $n(\%)$} \\
\hline Female & $174(88.3)$ & $86(88.7)$ \\
\hline Male & $23(11.7)$ & $11(11.3)$ \\
\hline \multicolumn{3}{|c|}{ Tumour location, $n(\%)$} \\
\hline Right & $120(60.9)$ & $53(54.6)$ \\
\hline Left & $77(39.1)$ & $44(45.4)$ \\
\hline \multicolumn{3}{|c|}{ Histological characteristics, $n(\%)$} \\
\hline Epithelioid & $153(77.7)$ & $79(81.4)$ \\
\hline Biphasic & $24(12.2)$ & $15(15.5)$ \\
\hline Sarcomatoid & $9(4.6)$ & $3(3.1)$ \\
\hline Mixed & $11(5.6)$ & \\
\hline
\end{tabular}

CMT: combined modality treatment; MPM: malignant pleural mesothelioma. 


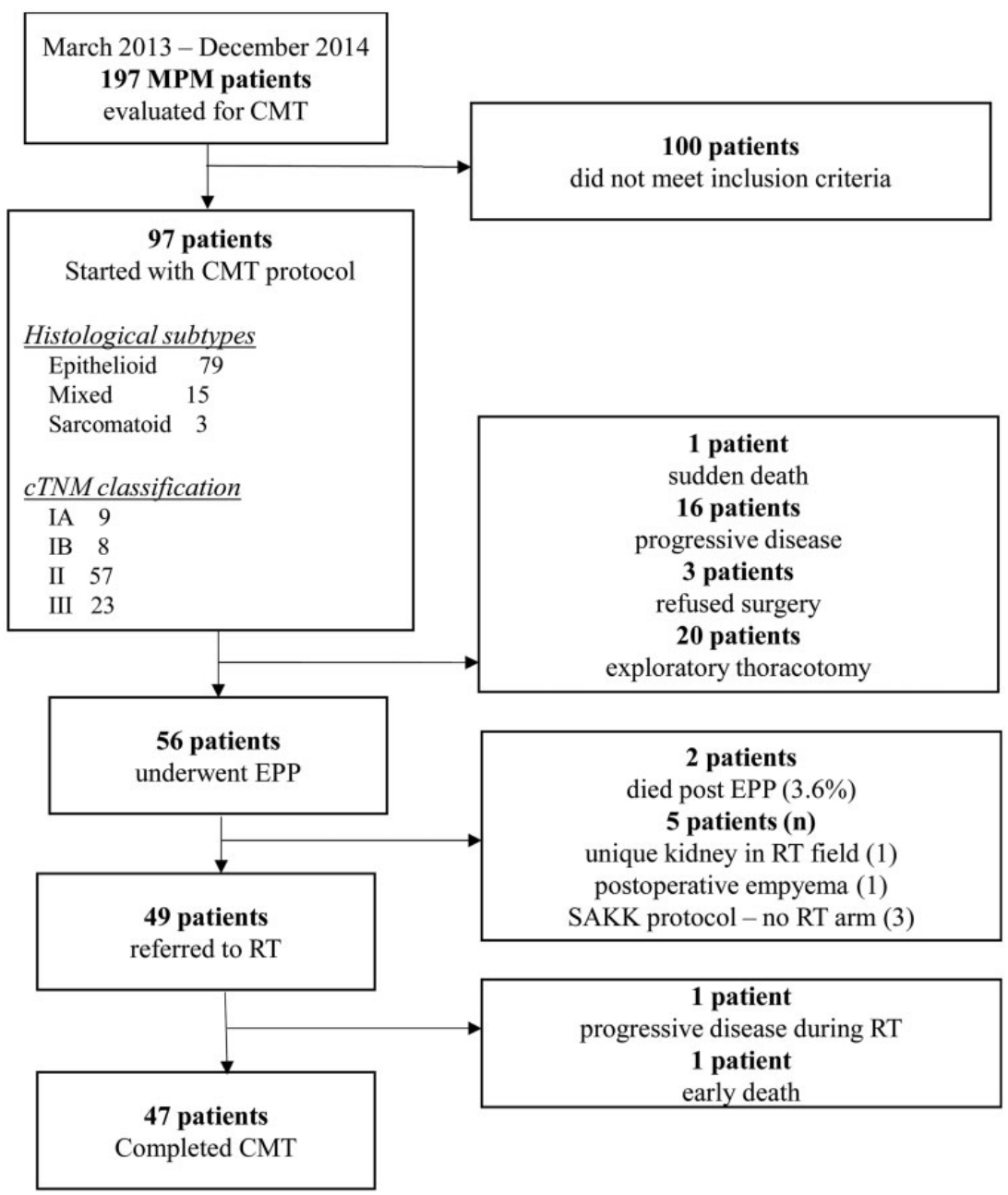

Figure 1: Schematic illustration of the total cohort with MPM. CMT: combined modality treatment; cTNM: clinical TNM staging [7th edition of the International Mesothelioma Interest Group (IMIG)/Union for International Cancer Control (UICC) staging system] [15]; EPP: extrapleural pneumonectomy; MPM: malignant pleural mesothelioma; RT: radiotherapy; SAKK: Swiss Group for Clinical Cancer Research.

Within this period, all patients presenting in our hospital with a biopsy-proven MPM were evaluated for inclusion in a combined modality protocol.

Inclusion criteria were MPM staging cT2N2M0 or less, all histological subtypes, age $\leq 70$ years, World Health Organization performance status $\leq 1$, medically fit for pneumonectomy partial pressure of carbon dioxide $\left(\mathrm{pCO}_{2}\right)<45 \mathrm{mmHg}$ and partial pressure of oxygen $\left(\mathrm{pO}_{2}\right)>65 \mathrm{mmHg}$ on air breathing, left ventricular ejection fraction calculated on cardiac ultrasound $>45 \%$ and predicted postoperative first second of forced expiration $\left(\mathrm{FEV}_{1}\right)>1 \mathrm{I}$ ) and results from kidney and liver function tests within normal ranges. Patients presenting with signs of chest wall invasion on chest computed tomography $(\mathrm{CT})$ or magnetic resonance imaging were excluded to increase the chance of achieving a macroscopically complete resection margin. Eligibility of candidates was discussed at the weekly meeting of the multidisciplinary tumour board.

\section{Pretreatment assessment}

All patients underwent a workup that included $\mathrm{CT}$ scans of the chest and abdomen followed by positron emission tomography (PET) or combined PET/CT scans and contrast-enhanced CT scans of the brain. Pulmonary evaluation consisted of bronchoscopy (not routinely), pulmonary function tests and quantitative ventilation-perfusion scintigraphy. Cardiac tests included electrocardiography and cardiac ultrasound. If, after this evaluation, the patient was deemed operable from oncological and medical points of view, video mediastinoscopy and laparoscopy (to exclude transmural invasion of the diaphragm) completed the pretreatment assessment. Thoracic magnetic resonance imaging or bone scintigraphy was not routinely performed.

\section{Induction chemotherapy}

Three cycles of chemotherapy that included cisplatin $\left(75 \mathrm{mg} / \mathrm{m}^{2}\right.$ day 1 every 3 weeks) and pemetrexed $\left(500 \mathrm{mg} / \mathrm{m}^{2}\right.$ day 1$)$ were given every 3 weeks, for a total of 3 cycles.

\section{Restaging after induction chemotherapy}

PET/CT or CT scans of the chest/abdomen were performed within 2 weeks after completion of induction chemotherapy (IC). An EPP was attempted when progressive disease (based on response evaluation criteria in solid tumours) was absent and the general condition of the patient did not preclude an EPP [12-14]. 


\section{Surgery}

All patients underwent general anaesthesia and double lumen intubation with the intention of performing EPP. A posterolateral thoracotomy with removal of the 6th rib was performed in every patient. Resectability was evaluated during extrapleural dissection. Chest wall or vertebral invasion, mediastinal invasion or transmural invasion of the diaphragm was considered irresectable. Otherwise, EPP was performed with en bloc removal of the pleura, ipsilateral lung, diaphragm and pericardium. Macroscopically complete resection was documented if no visible or palpable tumour was left. All other cases were deemed incompletely resected.

After removal of the resection specimen, the chest cavity was scrubbed with povidone-iodine sponges. Subsequently, the cavity was washed out with sterile water ( $3 \mathrm{I})$ and saline solution (3I) followed by hydrogen peroxide $(500 \mathrm{ml})$. The diaphragm was reconstructed with 2-mm thick GoreTex Dual Mesh ${ }^{\circledR}$; the pericardium was reconstructed using 1-mm thick GoreTex Dual Mesh. The thoracotomy was then closed after placement of a $28 \mathrm{Fr}$ chest drain in open drainage. Typically, the patient was kept in the intensive care unit for 2 days and generally extubated the night following the surgery. The chest drain was clamped and removed according to the results of the chest radiograph, usually 3 days to 4 days post-EPP.

\section{Hemithoracic radiation protocol}

The radiation treatment comprised $54 \mathrm{~Gy} / 1.8 \mathrm{~Gy}$ in 30 fractions over 6 weeks ( 5 fractions per week) to the entire hemilateral thoracic cavity, including all operative sites marked by buttons or clips. In 4 patients, an additional boost of $10 \mathrm{~Gy} / 2 \mathrm{~Gy}$ was delivered to the area of the microscopically positive or close section margin.

\section{Statistical analysis}

Overall survival (OS) was calculated from the time of diagnosis and analysed using a Kaplan-Meier survival plot. Survival differences were analysed with log-rank multiple tests. The Cox proportional hazards model was fitted to evaluate covariates that affected disease-free survival (DFS). $P$-values of $\leq 0.05$ were considered significant. All analyses were performed using SPSS version 24 (SPSS, Inc., Armonk, NY, USA).

\section{RESULTS}

From March 2003 to December 2014, 197 patients presenting with a MPM were discussed by the members of the multidisciplinary tumour board. The patient characteristics are outlined in Table 1 and in the consort diagram of the total MPM cohort shown in Fig. 1.

The main histological subtype of MPM was epithelioid ( $n=153,77.7 \%)$, followed by biphasic, desmoplastic and sarcomatoid subtypes.

Of the 197 patients initially diagnosed with MPM, 100 (50.8\%) did not fit the eligibility criteria for CMT after review during the weekly meeting of the multidisciplinary tumour board. The main reasons for exclusion were advanced stage $(n=49)$ and age $(n=40)$. In total, 97 patients fulfilled the inclusion criteria and were eligible for CMT. The histological subtypes of all 97 patients who received CMT are shown in Table 1. According to the 7th
Table 2: Complications during and after extrapleural pneumonectomy

\begin{tabular}{|c|c|}
\hline Complications $(n=56)$ & Patients, $n(\%)$ \\
\hline \multicolumn{2}{|l|}{ Pulmonary } \\
\hline Pulmonary infection & $3(5.4)$ \\
\hline Pulmonary insufficiency & $1(1.8)$ \\
\hline ARDS & $2(3.6)$ \\
\hline Pleural effusion contralateral side (no chest tube) & $2(3.6)$ \\
\hline \multicolumn{2}{|l|}{ Cardiac } \\
\hline Atrial fibrillation & $20(35.7)$ \\
\hline Dysrhythmia & $5(8.9)$ \\
\hline \multicolumn{2}{|l|}{ Neurological } \\
\hline Paralysis & $1(1.8)$ \\
\hline \multicolumn{2}{|l|}{ Thromboembolic } \\
\hline Deep venous thrombosis & $1(1.8)$ \\
\hline Pulmonary embolism & $1(1.8)$ \\
\hline \multicolumn{2}{|l|}{ Renal } \\
\hline Renal insufficiency & $3(5.4)$ \\
\hline Renal insufficiency requiring dialysis & $1(1.8)$ \\
\hline Other & $7(12.5)$ \\
\hline \multicolumn{2}{|l|}{ Surgical complications } \\
\hline Postoperative bleeding & $2(3.6)$ \\
\hline Chylothorax & $1(1.8)$ \\
\hline Empyema & $1(1.8)$ \\
\hline Wound infection & $1(1.8)$ \\
\hline
\end{tabular}

ARDS: acute respiratory distress syndrome.

edition of the International Mesothelioma Interest Group (IMIG)/ Union for International Cancer Control (UICC) staging system, the TNM staging of all 97 patients was stage IA $(n=9)$, IB $(n=8)$, II $(n=57)$ and III $(n=23)$ [15].

After IC, a complete response was observed in $2(2 \%)$, a partial response in $24(25 \%)$, stable disease in 56 (58\%) and progressive disease in 15 (15\%) patients. Twenty-one patients were not referred for surgery after IC for the reasons shown in Fig. 1.

A total of 76 patients underwent surgery. EPP was performed in 56 patients $(73.7 \%)$, and $20(26.3 \%)$ patients had an exploratory thoracotomy due to unresectable disease found during the procedure [chest wall invasion $(n=19)$ and oesophageal invasion $(n=1)$ ] that had not been identified preoperatively by imaging. Macroscopically complete resection was achieved in all patients who underwent $\operatorname{EPP}(n=57)$.

The median age in the EPP group was 57 years (range 35-70), and 7 patients $(12.5 \%)$ were women. After the final pathological examination of the 56 patients, 46 (82.1\%) had epithelioid subtype tumours, and 27 (48.2\%) had right-sided EPP. The median length of hospital stay was 13 days (range 8-55 days). Two patients (3.6\%) died during the postoperative hospitalization: 1 of multiorgan failure (due to respiratory infections) and 1 of postoperative acute respiratory distress syndrome.

The 30- and 90-day mortality rates were $0 \%(0 / 56)$ and $3.6 \%$ $(2 / 56)$, respectively.

The most frequently observed complications during and after EPP were classified into 7 groups: pulmonary, cardiac, neurological, thromboembolic, renal, surgical and other.

Surgical complications that we have observed in our patients during and after EPP are shown in Table 2. After EPP, 9.3\% (5/54) of the patients were not referred for radiotherapy (RT) whereas 2 patients (3.9\%) did not complete RT because of developing bone metastases in 1 and intercurrent respiratory failure in the other. 


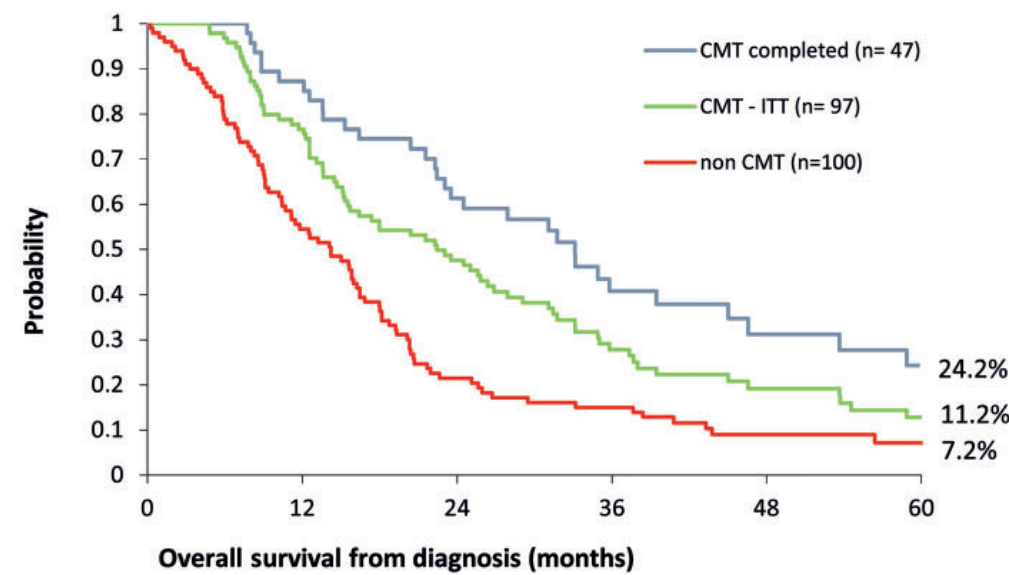

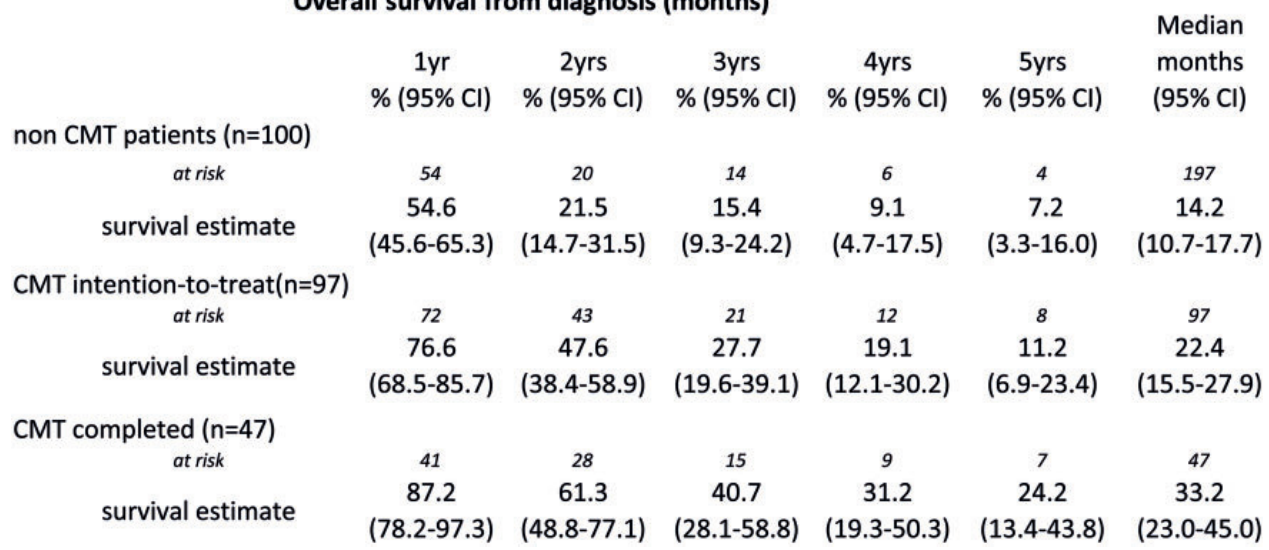

Figure 2: Overall survival from diagnosis (months) in patients who completed CMT ( $n=47$ ): the median OS was 33.2 months; the 1 -year survival rate was $87.2 \%$; and the 5 -year survival rate was $24.2 \%$. The median OS for the intent-to-treat CMT cohort $(n=97)$ was 22.4 months; the 1 -year survival rate was $76.6 \%$, and the 5 -year survival rate was $11.2 \%$. For the excluded patients $(n=100)$, the OS was 14.2 months; the 1 -year survival rate $54.6 \%$ and 5 -year survival rate was $7.2 \%$. Cl: confidence interval; CMT: combined modality treatment; ITT: intention to treat; OS: overall survival; pNO: no regional 'lymph node' metastases identified as positive.

In total, 47 patients received CMT and 5 patients received IC + EPP without RT. The intent-to-treat median and the 5-year OS rates were $22.4[95 \%$ confidence interval $(\mathrm{Cl})=15.5-27.9)$ months and $11.2 \%(95 \% \mathrm{Cl}=6.9-23.4)$. In patients who completed the CMT $(n=47)$, these values were $33.2(95 \% \mathrm{Cl}=23.0$ 45.0) months and $24.2 \%(95 \% \mathrm{Cl}=13.4-43.8)$. The intent-to-treat median and 5-year DFS were $15.6(95 \% \mathrm{Cl}=14.0-17.3)$ months and $9.9 \%(95 \% \mathrm{Cl}=5.1-19.2)$ and $19.8(95 \% \mathrm{Cl}=16.8-27.7)$ months and $17.2 \%(95 \% \mathrm{Cl}=8.6-34.1)$ in those with fully completed CMT (Figs 2 and 3). Cox regression analysis showed lymph node status after EPP to be the only significant factor to predict survival (Table 3$)$. A firm trend $(P=0.07)$ as an independent prognosticator for survival was observed for laterality (Table 3). In 30 (64\%) patients with epithelioid MPM who showed no positive lymph nodes ( $\mathrm{pN} 0$ ) after IC and EPP, the 5-year DFS was $27.0 \%$ (95\% Cl=14.1-51.7) (median 34.9 months, 95\% Cl=18.1-55.8) with EPP compared to $0 \%$ (median 15.8 months, $95 \% \mathrm{Cl}=11.3-$ $19.8)$ in 17 (36\%) patients with non-epithelioid tumours or who showed positive lymph nodes after EPP $(P=0.012)$ (Fig. 3).

\section{DISCUSSION}

Our single-centre cohort study describes the long-term outcome of patients with MPM who underwent CMT using EPP as a surgical procedure. We confirmed, for a group of well-selected patients with MPM, the feasibility of CMT with low rates of surgical morbidity and mortality. A 5 -year DFS of $27.0 \%$ (median 35 months) was observed in patients with epithelioid type MPM with negative lymph nodes ( $\mathrm{pN} 0$ ) after EPP, a result that is comparable to those from other centres performing EPP (Table 4). The multivariate analysis showed no significant difference in survival based on histological diagnosis $(P<0.06)$ due to the small size of the cohorts. Other studies showed similar results with epithelioid histological subtype and negative lymph node involvement being of prognostic significance in the multimodality therapy of MPM $[16,17]$.

The feasibility and safety of EPP for patients with MPM have been doubted, especially since the randomized MARS trial showed high rates of morbidity and mortality and a short median OS rate of 14.4 months (range 5.3-18.7 months) for the EPP arm [7].

Yet, despite the fact that the MARS trial was randomized, many have contested its conclusions because of methodological limitations and also because of the exceptionally high rates of morbidity (69\%) and mortality (12.5\% in-hospital) encountered in this study [8]. In contrast, several prospective series have shown the feasibility and safety of EPP-based multimodality treatment, with mortality rates consistently lower than $5 \%$ in experienced hands [18]. In a prospective, single-centre database, 529 patients with epithelioid MPM underwent complete resection by EPP in a multimodality strategy, with an in-hospital mortality rate limited to $5 \%$ (Table 3) [10].

Experience in performing EPP is of utmost importance to limit not only the number of deaths but also the number of 

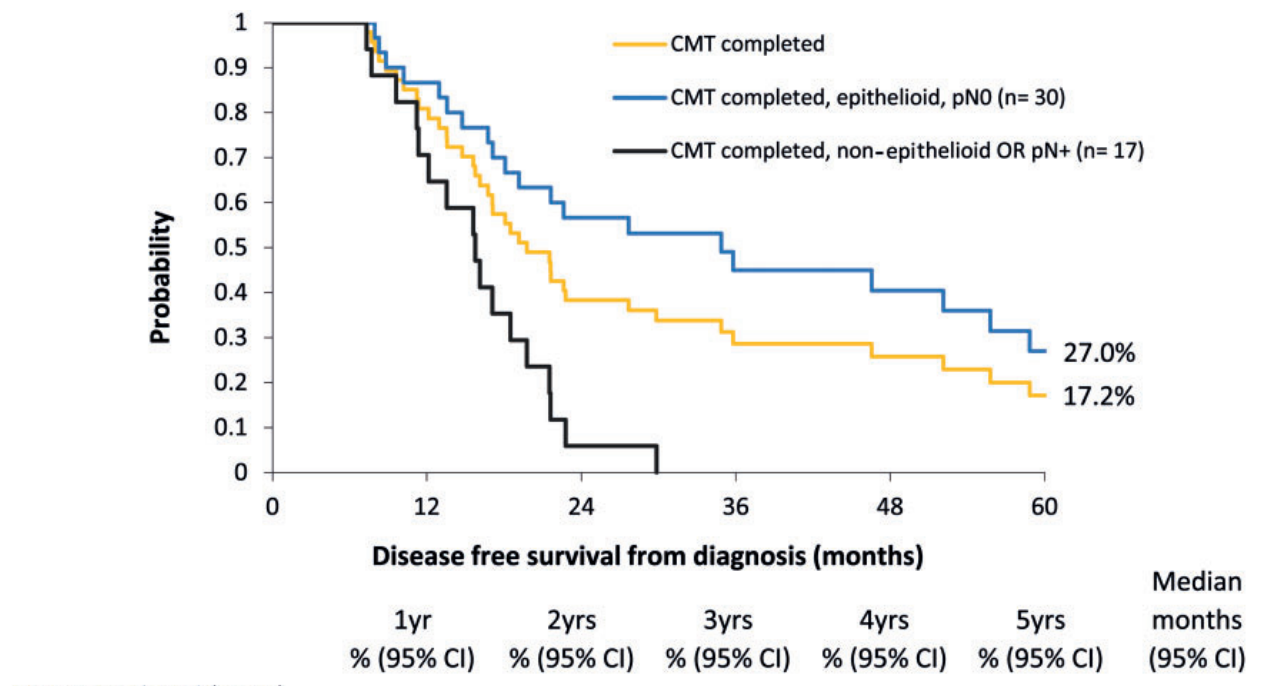

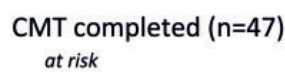

survival estimate

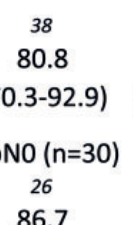

(75.3-99.7) $\begin{array}{cc}\text { CMT completed, epithelioid, } & \mathrm{pNO}(\mathrm{n}=30) \\ \text { at risk } & 26 \\ & 86.7\end{array}$

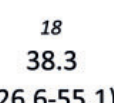

(26.6-55.1)
11

28.6

(18.0-45.5)

\section{7}

(15.5-42.8)

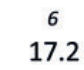

(8.6-34.1)
47

19.8

(16.8-27.7)

survival estimate

17
56.7

11

45.0

9

40.5

6
27.0

30

34.9

(18.1-55.8)

CMT completed, non-epithelioid OR pN+ (n=17)

at risk

12

5.9

0

0

0

17
15.8
$11.3-19.8$

Figure 3: DFS from diagnosis (months) in patients who completed CMT. The median DFS was 19.8 months (95\% Cl=16.8-27.7); the 1-year DFS was $80.8 \%$ (95\% $\mathrm{Cl}=70.3-92.9)$; and the 5-year DFS was $17.2 \%(95 \% \mathrm{Cl}=8.6-34.1)$ in those who fully completed the CMT. Patients with the epithelioid histological subtype and negative mediastinal lymph nodes had the best median DFS (34.9 months, 95\% Cl=18.1-55.8) and also the best 5-year DFS (27.0\%, 95\% Cl=14.1-51.7) compared to patients with a non-epithelioid subtype and positive lymph node involvement (median DFS: 15.8 months, $95 \% \mathrm{Cl}=11.3-19.8$, and 5-year DFS: $0 \%$ ). Cl: confidence interval; CMT: combined modality treatment; DFS: disease-free survival; pN+: positive regional lymph node metastasis; $\mathrm{pN0}$ : no regional 'lymph node' metastasis identified positive.

complications. After EPP, the most commonly observed medical complication is atrial fibrillation. Diaphragmatic patch dehiscence, chylothorax, haemothorax and empyema with or without bronchopleural fistula are the most common surgical complications [19].

In 63 patients who underwent complete EPP after neoadjuvant chemotherapy, Opitz et al. [20] observed post-pneumonectomy empyema in 10 patients (15.8\%) and a bronchopleural fistula in 6 patients. In a Swiss prospective multicentre trial, the aim was to evaluate the outcome, including quality of life, of IC followed by EPP and RT. The main complications observed were bronchopleural fistula and empyema in $62 \%$ of 45 patients undergoing EPP [21]. In our study, major surgical complications such as rebleeding, empyema and chylothorax were seen in only 5 patients (8.9\%). We did not observe bronchopleural fistula, and only 1 patient (1.8\%) developed a postoperative empyema. This finding can be explained by our surgical protocol of performing an extensive washout and using an intercostal muscle flap to protect the bronchial stump. In a large, prospective clinical database of 496 patients with a subset of 328 consecutive patients who underwent EPP, a bronchopleural fistula was also observed in only 2 patients $(0.6 \%)$ [22]. From 1980 to 2003, 496 consecutive patients underwent EPP at Brigham and Women's Hospital and the Dana Farber Cancer Institute, Boston, MA, USA. The incidence for empyema after EPP was 2.4\% (8 patients) [23]. Again, these results stress the importance of expertise when performing such a radical surgical procedure. Therefore, in our view and following recommendations [24], EPP should be limited to experienced centres within the boundaries of well-controlled studies or clearly defined clinical protocols performed by a multidisciplinary oncology team.

RT in patients with mesothelioma can be used for different indications such as palliation, prevention and as part of a multimodality treatment $[25,26]$.

Most of the patients (49/56) in our study who underwent and survived EPP were referred for adjuvant RT, in accordance with the latest guidelines of the European Society for Medical Oncology for MPM $[24,27]$. After EPP, adjuvant RT has been associated with improved disease control, a reduction of local recurrence and prolonged survival of patients with early-stage tumours [28]. Nevertheless, the safety and impact of post-EPP RT remains questionable and were evaluated in the SAKK 17/04 trial (Neoadjuvant Chemotherapy and Extrapleural Pneumonectomy of MPM With or Without Hemithoracic Radiotherapy) [29].

This randomized trial compared postoperative hemithoracic RT after neoadjuvant chemotherapy and EPP for MPM patients to the control group receiving the same treatment without RT. 
Table 3: Univariable and multivariable analyses of patients with malignant pleural mesothelioma with completed combined modality treatment

\begin{tabular}{|c|c|c|c|c|c|c|c|c|}
\hline & \multicolumn{4}{|c|}{ Univariable analysis } & \multicolumn{4}{|c|}{ Multivariable analysis } \\
\hline & \multirow[t]{2}{*}{ Sig. } & \multirow[t]{2}{*}{$\mathrm{HR}$} & \multicolumn{2}{|c|}{$95 \% \mathrm{Cl}$ for $\mathrm{HR}$} & \multirow[t]{2}{*}{ Sig. } & \multirow[t]{2}{*}{$\mathrm{HR}$} & \multicolumn{2}{|c|}{$95 \% \mathrm{Cl}$ for $\mathrm{HR}$} \\
\hline & & & Lower & Upper & & & Lower & Upper \\
\hline Age (years) & 0.226 & 1.022 & 0.986 & 1.060 & 0.564 & 1.011 & 0.974 & 1.050 \\
\hline Gender (male $/$ female) & 0.650 & 0.785 & 0.277 & 2.231 & 0.790 & 0.862 & 0.289 & 2.571 \\
\hline Performance status (fully active ${ }^{a} /$ restricted) & 0.801 & 0.921 & 0.487 & 1.744 & 0.858 & 0.939 & 0.475 & 1.859 \\
\hline Histological subtype (ep. ${ }^{a} /$ non-ep.) & 0.168 & 1.801 & 0.781 & 4.156 & 0.112 & 2.154 & 0.837 & 5.54 \\
\hline Laterality (right side ${ }^{a} /$ left side) & 0.073 & 0.562 & 0.300 & 1.054 & 0.369 & 0.703 & 0.326 & 1.517 \\
\hline Tract irradiation (yes $\mathrm{a} / \mathrm{no})$ & 0.657 & 0.923 & 0.649 & 1.313 & 0.611 & 0.797 & 0.331 & 1.915 \\
\hline $\mathrm{pT}<3^{\mathrm{a}} / \mathrm{pT} 3$ & 0.422 & 0.774 & 0.414 & 1.446 & 0.438 & 0.772 & 0.401 & 1.485 \\
\hline $\mathrm{pN}$ status ( $\mathrm{pN}$ negative $\mathrm{a} / \mathrm{pN}$ positive) & 0.006 & 2.790 & 1.346 & 5.782 & 0.022 & 2.699 & 1.151 & 6.326 \\
\hline Completed postoperative RTx (yes $/$ no) & 0.554 & 1.373 & 0.481 & 3.92 & 0.634 & 1.325 & 0.417 & 4.213 \\
\hline
\end{tabular}

Cox regression analysis showed lymph node status after extrapleural pneumonectomy to be the only significant factor to predict survival.

${ }^{\mathrm{a}}$ Reference.

Cl: confidence interval; ep.: epithelioid; HR: hazard ratio; pN: postoperative lymph node; pT3: pathological staging; RTx: radiotherapy; Sig.: significance.

Table 4: Comparison of the results (mortality and median OS) of extrapleural pneumonectomy as a surgical treatment in patients with malignant pleural mesothelioma

\begin{tabular}{|c|c|c|c|c|}
\hline Author (year) & Study design & $\operatorname{EPP}(n)$ & Mortality rate (\%) & $\begin{array}{l}\text { Median OS } \\
\text { (months) }\end{array}$ \\
\hline Weder et al. (2007) [21] & Prospective & 45 & 2.2 & 23 \\
\hline de Perrot et al. (2009) [31] & Retrospective & 45 & 6.7 & 14 \\
\hline Krug et al. (2009) [32] & Prospective & 54 & 3.7 & 16.8 \\
\hline Buduhan et al. (2009) [33] & Retrospective & 46 & 4.3 & 24 \\
\hline Van Schil et al. (2010) [5] & Prospective & 42 & 6.5 & 18.4 \\
\hline Federico et al. (2009) [34] & Retrospective & 41 & 4.4 & 15.5 \\
\hline Gomez et al. (2013) [35] & Retrospective & 136 & & 14.7 \\
\hline Sugarbaker et al. (2014) [10] & Prospective & 529 & 5 & 18 \\
\hline Spaggiari et al. (2014) [36] & Retrospective & 518 & 6.9 & 18 \\
\hline Stahel et al. (2015) [29] (SAKK 17/04 Trial) & Prospective & 113 & & 15 \\
\hline This study (2018) & Prospective & 56 & 3.6 & 33.1 \\
\hline
\end{tabular}

EPP: extrapleural pneumonectomy; OS: overall survival; SAKK: Swiss Group for Clinical Cancer Research.

Because the trial demonstrated that hemithoracic RT after EPP did not confer any clinical benefit, we also stopped using systematic postoperative RT as of 2015.

Besides the role of postoperative RT, IC is also debatable because the response to chemotherapy is limited and its use could potentially increase the risk of postoperative complications. These views have created an opportunity for other combinations of treatments that have already shown promising results $[10,11,25]$.

A weakness of our study was the retrospective character of all the analyses, which could obviously be the source of biases difficult to exclude in this setting. Further weaknesses are the small number of subgroups of patients with MPM in our analysis and the possible impact of an initial learning curve (after introduction of EPP in our centre) on our final results [30].

\section{CONCLUSION}

Our results demonstrated that patients with operable MPM had an acceptable survival and surgical mortality rate with CMT including IC and EPP, followed by adjuvant RT. Careful patient selection is of the highest importance because only half of the patients are able to complete CMT. Patients with epithelioid histological subtype and negative mediastinal lymph nodes had the best DFS and OS.

Conflict of interest: none declared.

\section{REFERENCES}

[1] Cancer Incidence in Belgium, 2004-2008. Belgian Cancer Registry. Brussels, 2012. https://kankerregister.org/media/docs/publications/ CancerSurvivalinBelgium.PDF

[2] Opitz I, Weder W. A nuanced view of extrapleural pneumonectomy for malignant pleural mesothelioma. Ann Transl Med 2017;5:237.

[3] Flores RM. Pleurectomy decortication for mesothelioma: the procedure of choice when possible. J Thorac Cardiovasc Surg 2016;151: $310-12$.

[4] Sugarbaker DJ, Flores RM, Jaklitsch MT, Richards WG, Strauss GM, Corson JM et al. Resection margins, extrapleural nodal status, and cell type determine postoperative long-term survival in trimodality therapy of malignant pleural mesothelioma: results in 183 patients. J Thorac Cardiovasc Surg 1999;117:54-63; discussion 63-5. 
[5] Van Schil PE, Baas P, Gaafar R, Maat AP, Van de Pol M, Hasan B et al. Trimodality therapy for malignant pleural mesothelioma: results from an EORTC phase II multicentre trial. Eur Respir J 2010;36: 1362-9.

[6] Schipper PH, Nichols FC, Thomse KM, Deschamps C, Cassivi SD, Allen MS et al. Malignant pleural mesothelioma: surgical management in 285 patients. Ann Thorac Surg 2008;85:257-64; discussion 64.

[7] Treasure T, Lang-Lazdunski L, Waller D, Bliss JM, Tan C, Entwisle J et al. Extra-pleural pneumonectomy versus no extra-pleural pneumonectomy for patients with malignant pleural mesothelioma: clinical outcomes of the Mesothelioma and Radical Surgery (MARS) randomised feasibility study. Lancet Oncol 2011;12:763-72.

[8] Weder W, Stahel RA, Baas P, Dafni U, de Perrot M, McCaughan BC et al. The MARS feasibility trial: conclusions not supported by data. Lancet Oncol 2011;12:1093-4; author reply 94-5.

[9] Cao C, Tian D, Park J, Allan J, Pataky KA, Yan TD. A systematic review and meta-analysis of surgical treatments for malignant pleural mesothelioma. Lung Cancer 2014;83:240-5.

[10] Sugarbaker DJ, Richards WG, Bueno R. Extrapleural pneumonectomy in the treatment of epithelioid malignant pleural mesothelioma: nove prognostic implications of combined $\mathrm{N} 1$ and $\mathrm{N} 2$ nodal involvement based on experience in 529 patients. Ann Surg 2014;260:577-80; discussion $80-2$.

[11] de Perrot M, Feld R, Leighl NB, Hope A, Waddell TK, Keshavjee S et al. Accelerated hemithoracic radiation followed by extrapleural pneumonectomy for malignant pleural mesothelioma. J Thorac Cardiovasc Surg 2016;151:468-73.

[12] Tsao AS, Garland L, Redman M, Kernstine K, Gandara D, Marom EM. A practical guide of the Southwest Oncology Group to measure malignant pleural mesothelioma tumors by RECIST and modified RECIST criteria. J Thorac Oncol 2011;6:598-601.

[13] Therasse P, Arbuck SG, Eisenhauer EA, Wanders J, Kaplan RS, Rubinstein $L$ et al. New guidelines to evaluate the response to treatment in solid tumors. European Organization for Research and Treatment of Cancer, National Cancer Institute of the United States, National Cancer Institute of Canada. J Natl Cancer Inst 2000;92:205-16.

[14] Eisenhauer EA, Therasse P, Bogaerts J, Schwartz LH, Sargent D, Ford R et al. New response evaluation criteria in solid tumours: revised RECIST guideline (version 1.1). Eur J Cancer 2009;45:228-47.

[15] Rusch VW. A proposed new international TNM staging system for malignant pleural mesothelioma. From the International Mesothelioma Interest Group. Chest 1995;108:1122-8.

[16] Meyerhoff RR, Yang CF, Speicher PJ, Gulack BC, Hartwig MG, D'Amico TA et al. Impact of mesothelioma histologic subtype on outcomes in the Surveillance, Epidemiology, and End Results database. J Surg Res 2015; 196:23-32.

[17] de Perrot M, Uy K, Anraku M, Tsao MS, Darling G, Waddell TK et al. Impact of lymph node metastasis on outcome after extrapleural pneumonectomy for malignant pleural mesothelioma. J Thorac Cardiovasc Surg 2007;133:111-16.

[18] Batirel HF. Extrapleural pneumonectomy (EPP). Ann Transl Med 2017;5: 232.

[19] Collaud S, de Perrot M. Technical pitfalls and solutions in extrapleural pneumonectomy. Ann Cardiothorac Surg 2012;1:537-43.

[20] Opitz I, Kestenholz P, Lardinois D, Müller M, Rousson V, Schneiter D et al. Incidence and management of complications after neoadjuvant chemotherapy followed by extrapleural pneumonectomy for malignant pleural mesothelioma. Eur J Cardiothorac Surg 2006;29:579-84.
[21] Weder W, Stahel RA, Bernhard J, Bodis S, Vogt P, Ballabeni P et al. Multicenter trial of neo-adjuvant chemotherapy followed by extrapleural pneumonectomy in malignant pleural mesothelioma. Ann Oncol 2007;18:1196-202.

[22] Sugarbaker DJ, Jaklitsch MT, Bueno R, Richards W, Lukanich J, Mentzer $\mathrm{SJ}$ et al. Prevention, early detection, and management of complications after 328 consecutive extrapleural pneumonectomies. J Thorac Cardiovasc Surg 2004;128:138-46.

[23] Zellos L, Jaklitsch MT, Al-Mourgi MA, Sugarbaker DJ. Complications of extrapleural pneumonectomy. Semin Thorac Cardiovasc Surg 2007;19: 355-9.

[24] Baas P, Fennell D, Kerr KM, Van Schil PE, Haas RL, Peters S et al. Malignant pleural mesothelioma: ESMO clinical practice guidelines for diagnosis, treatment and follow-up. Ann Oncol 2015;26 Suppl 5:v31-9.

[25] Opitz I. Management of malignant pleural mesothelioma-the European experience. J Thorac Dis 2014;6 Suppl 2:S238-52.

[26] Boutin C, Rey F, Viallat JR. Prevention of malignant seeding after invasive diagnostic procedures in patients with pleural mesothelioma. A randomized trial of local radiotherapy. Chest 1995;108:754-8.

[27] Stahel RA, Weder W, Lievens Y, Felip E; ESMO Guidelines Working Group. Malignant pleural mesothelioma: ESMO clinical practice guidelines for diagnosis, treatment and follow-up. Ann Oncol 2010;21 Suppl 5:v126-8.

[28] Rusch VW, Rosenzweig K, Venkatraman E, Leon L, Raben A, Harrison L et al. A phase II trial of surgical resection and adjuvant high-dose hemithoracic radiation for malignant pleural mesothelioma. J Thorac Cardiovasc Surg 2001;122:788-95.

[29] Stahel RA, Riesterer O, Xyrafas A, Opitz I, Beyeler M, Ochsenbein A et al. Neoadjuvant chemotherapy and extrapleural pneumonectomy of malignant pleural mesothelioma with or without hemithoracic radiotherapy (SAKK 17/04): a randomised, international, multicentre phase 2 trial. Lancet Oncol 2015;16:1651-8.

[30] Bovolato $P$, Casadio C, Billè A, Ardissone F, Santambrogio L, Ratto GB et al. Does surgery improve survival of patients with malignant pleural mesothelioma?: a multicenter retrospective analysis of 1365 consecutive patients. J Thorac Oncol 2014;9:390-6.

[31] de Perrot M, Feld R, Cho BC, Bezjak A, Anraku M, Burkes R et al. Trimodality therapy with induction chemotherapy followed by extrapleural pneumonectomy and adjuvant high-dose hemithoracic radiation for malignant pleural mesothelioma. J Clin Oncol 2009;27:1413-18.

[32] Krug LM, Pass HI, Rusch VW, Kindler HL, Sugarbaker DJ, Rosenzweig KE et al. Multicenter phase II trial of neoadjuvant pemetrexed plus cisplatin followed by extrapleural pneumonectomy and radiation for malignant pleural mesothelioma. J Clin Oncol 2009;27:3007-13.

[33] Buduhan G, Menon S, Aye R, Louie B, Mehta V, Vallières E. Trimodality therapy for malignant pleural mesothelioma. Ann Thorac Surg 2009;88: 870-5; discussion 76

[34] Federico R, Adolfo F, Giuseppe M, Lorenzo S, Martino DT, Anna C et al. Phase II trial of neoadjuvant pemetrexed plus cisplatin followed by surgery and radiation in the treatment of pleural mesothelioma. BMC Cancer 2013;13:22.

[35] Gomez DR, Hong DS, Allen PK, Welsh JS, Mehran RJ, Tsao AS et al. Patterns of failure, toxicity, and survival after extrapleural pneumonectomy and hemithoracic intensity-modulated radiation therapy for malignant pleural mesothelioma. J Thorac Oncol 2013;8:238-45.

[36] Spaggiari L, Marulli G, Bovolato P, Alloisio M, Pagan V, Oliaro A et al. Extrapleural pneumonectomy for malignant mesothelioma: an Italian multicenter retrospective study. Ann Thorac Surg 2014;97:1859-65. 